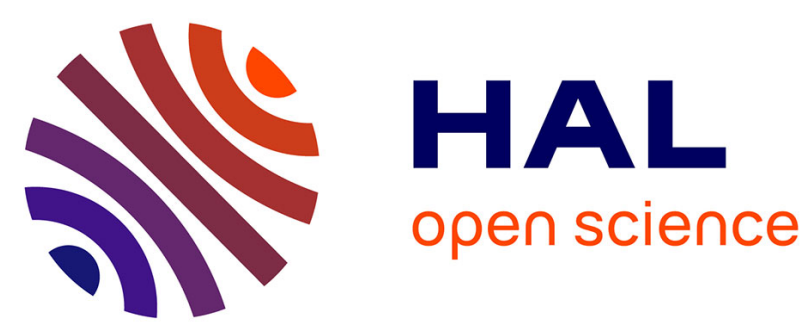

\title{
LaMgX and CeMgX (X = Ga, In, Tl, Pd, Ag, Pt, Au) with ZrNiAl type structure - A systematic view on electronic structure and chemical bonding
}

Samir F. Matar, Jean Etourneau, Rainer Pöttgen

\section{- To cite this version:}

Samir F. Matar, Jean Etourneau, Rainer Pöttgen. LaMgX and CeMgX (X = Ga, In, Tl, Pd, Ag, Pt, $\mathrm{Au}$ ) with $\mathrm{ZrNiAl}$ type structure - A systematic view on electronic structure and chemical bonding. Solid State Sciences, 2015, 43, pp.28-34. 10.1016/j.solidstatesciences.2015.03.017 . hal-01138723

\section{HAL Id: hal-01138723 \\ https://hal.science/hal-01138723}

Submitted on 2 Apr 2015

HAL is a multi-disciplinary open access archive for the deposit and dissemination of scientific research documents, whether they are published or not. The documents may come from teaching and research institutions in France or abroad, or from public or private research centers.
L'archive ouverte pluridisciplinaire HAL, est destinée au dépôt et à la diffusion de documents scientifiques de niveau recherche, publiés ou non, émanant des établissements d'enseignement et de recherche français ou étrangers, des laboratoires publics ou privés.

\section{(ㅇ)(1) $\$$}

Distributed under a Creative Commons Attribution - NonCommercial - NoDerivatives $\mid 4.0$ 
$\operatorname{LaMg} X$ and $\operatorname{CeMg} X(X=\mathrm{Ga}, \mathrm{In}, \mathrm{Tl}, \mathrm{Pd}, \mathrm{Ag}, \mathrm{Pt}, \mathrm{Au})$ with $\mathrm{ZrNiAl}$ type structure - A systematic view on electronic structure and chemical bonding

Samir F. Matar ${ }^{\mathrm{a}, *}$, Jean Etourneau ${ }^{\mathrm{a}}$, Rainer Pöttgen ${ }^{\mathrm{b}}$,

a CNRS, Université de Bordeaux, ICMCB, 87 Avenue du Docteur Albert Schweitzer, 33600 Pessac, France

$b$ Institut für Anorganische und Analytische Chemie, Universität Münster, Corrensstrasse 30, D-48149 Münster, Germany

\section{A R T I C LE IN F O}

\section{Article history:}

Received

Accepted

Available online \#\#\#\#

\section{A B S T R A C T}

The intermetallic magnesium compounds $\operatorname{LaMg} X$ and $\operatorname{CeMg} X(X=\mathrm{Ga}, \mathrm{In}, \mathrm{Tl}, \mathrm{Pd}$, $\mathrm{Ag}, \mathrm{Pt}, \mathrm{Au}$ ) crystallize with the hexagonal $\mathrm{ZrNiAl}$ type structure, space group $P \overline{6} 2 \mathrm{~m}$, with full $\mathrm{Mg}-X$ ordering. From density functional theory calculations carried out exemplarily on four representative compounds: $\operatorname{LaMg} X$ and $\operatorname{CeMg} X$ with $X=\mathrm{Ga}, \mathrm{Pd}$, significant differences were traced out as to the magnetism arising only for the Ce series leading to identify $\mathrm{CeMgGa}$ as an antiferromagnet in its ground state, in agreement with experiment. The bulk module magnitudes show the trend of harder transition metal based ternaries and the cohesive energies favor the $X=\mathrm{Pd}$ compounds versus $X=\mathrm{Ga}$ ones. Such features were clarified by examining the properties of chemical bonding which exhibit more directional bonds thanks to the $\operatorname{Pd} d$ states. Rationalizing the trends of charge transfers, negatively charged triel and transition element atoms are observed. The resulting chemical pictures assign these compounds as gallides and palladides. 
Keywords: A. functional alloys (magnetic, electrical, biomedical); B. Magnetic properties; B. Density functional theory; E. Ab initio calculations

(C) 2015 Elsevier Ltd. All rights reserved.

* Corresponding author. Fax: +33-540-00-2761.

E-mail address: Samir.Matar@icmcb.cnrs.fr \& abouliess@gmail.com (S.F. Matar).

\section{Introduction}

The equiatomic CeTX ( $T=$ electron-rich transition metal; $X=$ element of the $3^{\text {rd }}, 4^{\text {th }}$, or $5^{\text {th }}$ main group) phases [1] are one of the broadest investigated families of intermetallic rare earth compounds due to their largely differing magnetic and electric properties. Some outstanding examples are the heavy-fermion silicide CePtSi [2], the $10 \mathrm{~K}$ ferromagnet $\mathrm{CeAuGe}$ [3], the formation of an anisotropic energy gap in the valencefluctuating stannide CeNiSn [4], the Kondo semiconductor CeRhSb [5, 6], the nonFermi liquid system CeRhSn [7], the valence modulations in CeRuSn [8, 9], or the heavy-fermion $\left(\gamma=300 \mathrm{~mJ} /\left(\mathrm{mol} \mathrm{K}^{2}\right)\right)$ antiferromagnet $\left(\mathrm{T}_{\mathrm{N}}=0.9 \mathrm{~K}\right) \mathrm{CePtPb}[10]$. Several hundred manuscripts on $\mathrm{Ce} T X$ intermetallics have been published. Most data are summarized in review articles $[11,12]$.

The peculiar bonding characteristics and magnetic ground states of the CeTX intermetallics are often a consequence of the Ce- $T$ hybridization. Substitution of the $X$ component or the formation of solid solutions on both the $T$ and $X$ sites leads to substantial influence on the cerium electronic situation and thus changes in the magnetic behavior, e. g. $\mathrm{CePt}_{1-x} \mathrm{Ni}_{x} \mathrm{Sn}$ with a transition from an antiferromagnetic Kondo lattice to a mixed valence semiconductor [13], $\mathrm{CeNi}_{1-\mathrm{x}} \mathrm{Rh}_{\mathrm{x}} \mathrm{Sn}$ with an interplay between spin-glass-like and non-Fermi-liquid behavior [14] or $\mathrm{CeRhSb}_{1-\mathrm{x}} \mathrm{Sn}_{\mathrm{x}}$ with a quantum criticality at the border of Kondo insulator / non-Fermi liquid [7].

Other parameters that influence the magnetic behavior are temperature, highpressure or hydrogenation. Recent examples are the low- and high-temperature modifications of CePdZn [15] with stronger Ce(4f)-Pd(4d) hybridization in $\alpha-C e P d Z n$, the temperature induced structural and cerium valence change in CeRhGe [16] and the 
high-pressure modification of CePtSn [17]. Hydrogenation of CeZnSn to $\mathrm{CeZnSnH}_{1.5}$ increases the Curie temperature from 4.8 to $7.3 \mathrm{~K}$ [18].

Modification of the magnetic properties is also possible through substitution of the transition metal by another $p$ element, thus suppressing $d-f$ hybridization. First investigations in such phase diagrams led to the magnetically ordering phases CeGaGe $[19$, 20], CeAlSi and CeAlGe [21] and CeGaSi [22, 23]. Systematic studies have been performed for the $R E \mathrm{Mg} X$ ( $X=\mathrm{Ga}, \mathrm{In}, \mathrm{Tl})$ phases [24-28] with $\mathrm{ZrNiAl}$ type structure, including the cerium intermetallics $\mathrm{CeMgGa}, \mathrm{CeMgIn}$, and $\mathrm{CeMgTl}$ of which $\mathrm{CeMgGa}$ orders antiferromagnetically at $\mathrm{T}_{\mathrm{N}}=3.1 \mathrm{~K}$. These phases can be considered as the $p$ element analogues of the transition metal counterparts $\mathrm{CeMg} T$ ( $T=\mathrm{Pd}, \mathrm{Ag}, \mathrm{Pt}, \mathrm{Au})$ [2931]. These substitutions offer an interesting situation for changes in chemical bonding while keeping the structure type. Herein we present a detailed study of the electronic structures and the chemical bonding situation of these two families of intermetallics.

\section{Computational details}

Within the framework of the density functional theory (DFT) $[32,33]$ two computational methods were used in a complementary manner. For the purpose of firstly obtaining energy (total and cohesive) and charge transfer quantities, the Vienna a $b$ initio simulation package (VASP) code [34, 35] was used with the projector augmented wave (PAW) method [35, 36]. DFT exchange and correlation effects were accounted for in the framework of the generalized gradient approximation (GGA) scheme following Perdew, Burke and Ernzerhof (PBE) [37]. Atoms were relaxed and the conjugategradient algorithm [38] is used in this computational scheme. The tetrahedron method with Blöchl corrections [39] as well as Methfessel-Paxton [40] Brillouin-zone (BZ) integrals were approximated using the special $k$-point sampling of Monkhorst and Pack [41]. A large number of $150 \mathrm{k}$-points was used in the irreducible wedges of the hexagonal Brillouin zone to perform total energy calculations. The optimization of the structural parameters was performed until the forces on the atoms were less than $0.02 \mathrm{eV}^{-1}$ and all stress components less than $0.003 \mathrm{eV}^{-3}$.

Then the full investigation of the electronic and magnetic structures and the chemical bonding properties was performed using the full potential augmented spherical wave (ASW) method [42, 43] in a scalar relativistic implementation [44] with exchange- 
correlation effects accounted for within the generalized gradient approximation GGA [37]. In the ASW method, the wave function is expanded in atom-centered augmented spherical waves, which are Hankel functions and numerical solutions of Schrödinger's equation, respectively, outside and inside the so-called augmentation spheres. In the minimal ASW basis set, we chose the outermost shells to represent the valence states and the matrix elements were constructed using partial waves up to $l_{\max }+1=4$ for La and Ce, $l_{\max }+1=3$ for Pd and, $l_{\max }+1=2$ for Mg and Ga. Self-consistency was achieved when charge transfers and energy changes between two successive cycles were such as: $\Delta \mathrm{Q}<10^{-8}$ and $\Delta \mathrm{E}<10^{-6} \mathrm{eV}$, respectively. The BZ integrations were performed using the linear tetrahedron method [39] within the irreducible hexagonal wedge.

The calculations were carried out assuming firstly spin degenerate, non spinpolarized (NSP), then spin polarized (SP) magnetic calculations for both ferromagnetic and antiferromagnetic long range orders for the cerium compounds. The relative magnitude of the chemical bonding is obtained based on the overlap population analysis: $S_{\mathrm{ij}}, \mathrm{i}$ and $\mathrm{j}$ being two chemical species. The crystal orbital overlap population (COOP) criterion is used [45]. In the plots positive, negative and zero COOP magnitudes indicate bonding, anti-bonding and non-bonding interactions respectively.

\section{Results and discussion}

\subsection{Structural chemistry}

The $\operatorname{CeMg} X(X=\mathrm{Ga}, \mathrm{In}, \mathrm{Tl}, \mathrm{Pd}, \mathrm{Ag}, \mathrm{Pt}, \mathrm{Au})$ phases crystallize with the hexagonal ZrNiAl type structure [46-48], space group $P \overline{6} 2 m$ Pearson code hP9, and Wyckoff sequence $g f d a$. A projection of the $\operatorname{CeMg} X$ structure onto the $x y$ plane is presented in Fig. 1. The striking structural motif is the trigonal prismatic coordination of the $X$ atoms. The $X 1$ atoms are coordinated by six cerium atoms and six of these $X 1 @ \mathrm{Ce}_{6}$ prisms condense via common edges forming hexagonal rings. The latter are filled by the $X 2 @ \mathrm{Mg}_{6}$ prisms. Both prismatic motifs are shifted with respect to each other by half the $c$ lattice parameter, emphasized by thin and thick lines in Fig. 1. Condensation along the $c$ axis proceeds through common triangular faces. 
The cell parameters of the $\operatorname{CeMgX}$ phases are listed in Table 1 along with their lanthanum counterparts. The latter have been used for additional calculations in order to avoid problems with the $f$ states. For all pairs of compounds the cell volume of the cerium phase is smaller, a consequence of the lanthanide contraction. From comparison with the complete series [25-27, 29], all cerium phases are trivalent, in agreement with the magnetic susceptibility data. An interesting situation occurs for the compounds with the heaviest $X$ components. In the pairs $\mathrm{CeMgPd} / \mathrm{CeMgPt}, \mathrm{CeMgAg} / \mathrm{CeMgAu}$, and $\mathrm{CeMgIn/CeMgTl,} \mathrm{the} \mathrm{platinum,} \mathrm{gold,} \mathrm{and} \mathrm{thallium} \mathrm{compound} \mathrm{show} \mathrm{the} \mathrm{smaller} \mathrm{cell}$ volumes, a consequence of the relativistic contraction.

A drastic difference between the $\operatorname{CeMgX}$ phases with a $p$ and a $d$ element is the much larger c/a ratio for those with the $p$ elements (Table 1). This behavior is directly related to the magnesium triangles forming the $X 2 @ \mathrm{Mg}_{6}$ trigonal prisms around the origin of the unit cells. The Mg-Mg distances for CeMgIn (325.9 pm), CeMgTl (327.0 pm), CeMgAg (325.6 pm), and CeMgAu (323.1 pm) are all close to the average $\mathrm{Mg}-$ $\mathrm{Mg}$ distance of $320 \mathrm{pm}$ in hcp magnesium [49]. Thus, changes in $\mathrm{Mg}-X$ bonding are managed through increase or decrease of the height of the trigonal prism.

The shortest interatomic distances in the $\operatorname{CeMg} X$ structures occur for $\mathrm{Mg}-X$. The average Mg-In (302 pm), Mg-Tl (301 pm), Mg-Ag (295 pm), and Mg-Au (291 pm) distances (Table 2) are all longer than the sums of the covalent radii [50] of 286, 291, 270, and $270 \mathrm{pm}$, respectively, indicating medium bond strength. It is evident from Table 2, that the cerium atoms have five indium, thallium, silver, or gold atoms as nearest neighbors. Thus, the electropositive cerium atoms bind to the more electronegative triel or transition metal atoms. Accordingly we can ascribe thallide and auride character to $\mathrm{CeMgTl}$ and $\mathrm{CeMgAu}$. These features of chemical bonding are addressed in more detail in the following sections.

\subsection{Electronic structure and chemical bonding}

In view of the large $\operatorname{CeMg} X$ and $\operatorname{LaMg} X$ equiatomic family of $X=$ triel and $X=$ transition metal intermetallics, we have elected to show the results representative of $X=\mathrm{Ga}$ and $X=\mathrm{Pd}$ respectively.

\subsubsection{Energies and derived quantities}


The experimental crystal data (Table 1) were firstly used for carrying out unconstrained geometry optimization runs with VASP-PAW-GGA in order to get the minimum equilibrium energies and energy derived quantities as the bulk modulus $\mathrm{B}_{0}$ based on Birch energy-volume equation of state [51] for the four selected compounds, as well as an analysis of trends of charge transfers. Table 3 provides the calculated parameters of total electronic energy, cohesive energy, volume and $\mathrm{B}_{0}$. According to the nature of $X$, the compounds are regrouped in close values of all four quantities with smaller magnitudes for $X=\mathrm{Ga}$. The calculated volumes are found in relative good agreement with experiment with the trend of larger values due to the use of the GGA XC functional known to be under-binding. The cohesive energies obtained by subtracting the energies of the atomic constituents in their ground states from the total energies. The $X=\mathrm{Pd}$ compounds are found more cohesive than $X=\mathrm{Ga}$ ones and the cerium based compounds are found with larger cohesive energy than the lanthanum compounds Similar trends are found for the bulk modules which are found larger for $X=\mathrm{Pd}$ than for $X=\mathrm{Ga}$ with larger magnitudes for the Ce based ternaries. With one more electron brought in by Ce versus La and the presence of $d$ states in Pd versus $p$ Ga states, directional bonding should be at the origin of the observed differences. These aspects can be reflected in the analysis of the chemical bonding discussed in the next section. Lastly the $\mathrm{B}_{0}$ magnitudes are within the range of ternary equiatomic intermetallic compounds [11].

We also discuss the trends of charge transfers obtained from the analysis of the charge density within the atoms in molecules AIM theory of Bader [52]. Table 4 provides the charges on the respective atoms in the four studied compounds. The trend of negatively charged $X$ is observed in all four compounds leading to assign the chemical labeling of gallides and palladides. Positively charged $\mathrm{La}, \mathrm{Ce}$ and $\mathrm{Mg}$ are identified with larger electropositive values in the Pd based intermetallics likely due to the larger electronegativity: $\chi \mathrm{Pd}>\chi \mathrm{Ga}(2.2$ versus 1.81$)$ while $\mathrm{Ce}$ and La have close $\chi$ of 1.1 . The difference in magnitudes between $X 1$ and $X 2$ arises from their respective crystal chemistry, i.e. with respect to $X 1 @ \mathrm{Ce}_{6}$ prisms versus $X 2 @ \mathrm{Mg}_{6}$ prisms.

3.2.2 Total-spins and spin-polarized magnetic calculations (NSP, SP) 
With the experimental structure parameters (Table 1) the complete site and spin projected density of states (DOS) were computed in both non spin polarized (NSP) and spin polarized (SP) configurations using the full potential scalar relativistic ASW method.

Figs. 2 and 3 regroup the NSP DOS for the two series. Along the $x$ axis the energy reference is with respect to the Fermi level $\left(\mathrm{E}_{\mathrm{F}}\right)$ which is crossed by a large $\mathrm{Ce}(\mathrm{DOS})$ while a deep minimum is observed for $\mathrm{LaMgGa}$ and a small DOS magnitude can be seen for $\mathrm{LaMgPd}$. The difference is due to the electron configuration of the rare earth elements $\mathrm{Ce}$ and $\mathrm{La}$ which differ by one $4 f$ electron. This point is further stressed in the developments related with the magnetic instability.

The valence band -VB- shows large differences due to the $\mathrm{Pd} d$ states at $\sim-3.5 \mathrm{eV}$ in Fig. 3 whereas small magnitude broad Ga $p$ states are observed in the VB of Fig. 2. In so far that the bonding is mainly ensured within the VB, one may expect different bonding behaviors as already suggested in the preceding section.

Empty La $4 f$ states are mainly found above $\mathrm{E}_{\mathrm{F}}$ within the conduction band - $\mathrm{CB}-$ where the low occupied Ce $4 f$ band is centered. The large magnitude of the Ce DOS at EF signals instability of the Ce compounds in such a NSP configuration, meaning that stabilization should incur if a spin polarized SP configuration is considered. Subsequent SP calculations lead, assuming implicitly a ferromagnetic configuration (SPF), indeed to the stabilization of the $\mathrm{Ce}$ based compounds and the onset of finite magnetic moments.

Table 5 provides the values of energy differences between the considered magnetic configurations and the spin only magnetic moment on Ce. For CeMgGa the energy difference between the two magnetic configurations is $\Delta E_{\mathrm{SPF}-\mathrm{NSP}}=-0.172 \mathrm{eV} / \mathrm{cell}$. This value deceases down to $-0.046 \mathrm{eV}$ for $X=\mathrm{Pd}$. The $\mathrm{SPF}$ configuration is stabilized with respect to NSP due to magnetic exchange which is then larger magnetic $X=\mathrm{Ga}$ case leading to larger magnitude of the Ce moment. Small moments are obtained for the other constituents resulting from some residual polarization due to the mixing of the valence states with those of magnetically polarized $\mathrm{Ce}$. In view of the fact that both $\mathrm{CeMg} X$ order antiferromagnetically at $\mathrm{T}_{\mathrm{N}}=3.12 .1 \mathrm{~K}$ respectively [30], further SP antiferromagnetic (SPAF) calculations were carried out by doubling the cell along $c$ so that the atoms belonging to the first half of the double cell are assigned SPIN UP magnetic 
subcell and the other half SPIN DOWN magnetic subcell. For CeMgGa the calculations lead to further stabilization $\Delta E_{\mathrm{SPAF}-\mathrm{SPF}}=-0.04 \mathrm{eV} / \mathrm{cell}$ whereas similar SPAF calculations carried on CeMgPd led to a vanishing of the magnetization. This can be assigned to its smaller $T_{N}$ value together with the lower magnitudes of the magnetization in the SPF configuration with respect to CeMgGa. This likely leads to instability of the heavier SPAF calculations with subsequent vanishing magnetic polarization on the atomic constituents, mainly Ce.

The SPF and SPAF results are further illustrated with the site and spin projected DOS shown in Fig. 4.

In the first two panels corresponding to SPF calculations, the majority spin $(\uparrow)$ are shifted to lower energy versus minority spin states $(\downarrow)$. This energy difference, related to magnetic exchange, leads to the onset of the magnetic moment which is, as described above, carried only by Ce. Within the valence bands of both compounds, very small intensity $\mathrm{Ga}, \mathrm{Pd} d$ states and itinerant $\mathrm{Mg}$ and $\mathrm{Ce}$ states mix in a similar way as in the NSP state without any energy shift between the two spin channels ( $\uparrow$ and $\downarrow$ ). For Ce, it can be noted the larger energy shift in $\mathrm{CeMgGa}$ as with respect to $\mathrm{CeMgPd}$, proportional to the magnitudes of the respective magnetic moments and total magnetizations (Table 5). The third panel of Fig. 4 shows the fully compensated magnetizations between the two magnetic subcells 'SPIN UP' and 'SPIN DOWN', signature of the SPAF configuration of $\mathrm{CeMgGa}$.

\subsubsection{Properties of chemical bonding (COOP)}

Through exploring the energy quantities (Table 3) it was found out that the Pd based intermetallics were significantly more cohesive versus the Ga ones. This is also accompanied by larger hardness. These observations let suggest a role played by the chemical bonding accounting for pair interactions. Such properties can be qualitatively described based on the analysis of the overlap integrals $S_{i j}$ within the crystal orbital overlap population (COOP) introduced by Hoffmann in quantum chemical methods [45] and implemented into the ASW method [44].

Figure 5 shows the COOP for $X=\mathrm{Ga}$ (top panels) and $X=\mathrm{Pd}$ (bottom panels). The calculations are for NSP configuration. Whereas in all panels the VB is of positive CO- 
OP intensities, i.e. of bonding nature, the antibonding states starts developing above $\mathrm{E}_{\mathrm{F}}$ within the CB. This comforts the cohesive nature of all compounds (Table 3).

Differences can be observed at two different levels, pertaining:

1) to the nature of $X \mathrm{~d}$ which binds either with $p$ states $(\mathrm{Ga})$ in the close neighborhood of $\mathrm{EF}_{\mathrm{F}}$ or with more intense and localized (directional) $d$ states (Pd) at -4 $\mathrm{eV}$ below $\mathrm{E}_{\mathrm{F}}$; and

2) to the nature of the $4 f$ element La or Ce whereby the latter has bonding sharp $\mathrm{COOP}$ at $\mathrm{EF}_{\mathrm{F}}$ whereas La shows broader COOP arising mainly from $d$ states, a character missing with the Ga itinerant states.

The major bonding contribution arising from ( $\mathrm{La}, \mathrm{Ce})-\mathrm{Ga}$ and $(\mathrm{La}, \mathrm{Ce})-\mathrm{Pd}$ and the latter being more directional one may understand the larger bulk modulus of the Pd based compounds (Table 1).

\section{Conclusions}

In this work aiming at better rationalizing the properties of a rich family of $\mathrm{Mg}$ based intermetallic compounds: $\operatorname{LaMg} X$ and $\operatorname{CeMg} X$ ( $X=$ triel or transition metal), DFT-built methods were applied to four representatives based on $\mathrm{Ga}$ and Pd. From charge transfer trends, the compounds are found as 'gallides' and 'palladides' with stronger cohesion and larger hardness characterizing the latter family. Magnetic polarization is found only in the Ce based compounds with a calculated antiferromagnetic ground state in agreement with experiment. Qualitative chemical bonding analysis shows different behaviors between the $\mathrm{Ga}$ and $\mathrm{Pd}$ families with a role played by Pd assigned to directional $d$ states versus Ga broad $s, p$ and subsequent stronger bonding for the former. This further illustrates the calculated cohesive energy and bulk module trends.

\section{Acknowledgment}

This work was financially supported by the Deutsche Forschungsgemeinschaft. We acknowledge support from the Conseil Régional d'Aquitaine. Part of the computations were carried out on the MCIA supercomputers of the University of Bordeaux. 


\section{References}

[1] Villars P, Cenzual K. Pearson's Crystal Data: Crystal Structure Database for Inorganic Compounds (release 2014/15), ASM International@, Materials Park, Ohio (USA) 2014.

[2] Lee WH, Shelton RN. CePtSi: A New Heavy-Fermion Compound. Phys Rev B 1987;35:5369-5371.

[3] Pöttgen R, Borrmann H, Kremer RK. Ferromagnetic Ordering in CeAuGe. J Magn Magn Mater 1996;152:196-200.

[4] Takabatake T, Teshima F, Fujii H, Nishigori S, Suzuki T, Fujita T, Yamaguchi Y, Sakurai J, Jaccard D. Formation of an Anisotropic Energy Gap in the ValenceFluctuating System CeNiSn. Phys Rev B 1990;41:9607-9610.

[5] Malik SK, Adroja DT. Evidence of Pseudogap Formation in a New ValenceFluctuating Compound: CeRhSb. Phys Rev B 1991;43:6277-6279.

[6] Chevalier B, Decourt R, Heying B, Schappacher FM, Rodewald UCh, Hoffmann R-D, Pöttgen R, Eger R, Simon A. Inducing Magnetism in the Kondo Semiconductor CeRhSb through Hydrogenation - Antiferromagnetic Behaviour of the New Hydride CeRhSbH0.2. Chem Mater 2007;19:28-35.

[7] Ślebarski A. Low-Temperature Anomalies in Magnetic, Electric Transport and Thermal Properties of CeRhSn. J Low Temp Phys 2007;147:147-163.

[8] Riecken JF, Hermes W, Chevalier B, Hoffmann R-D, Schappacher FM, Pöttgen R. Trivalent-Intermediate Valent Cerium Ordering in CeRuSn - A Static Intermediate Valent Cerium Compound with a Superstructure of the CeCoAl Type. Z Anorg Allg Chem 2007;633:1094-1099.

[9] Feyerherm R, Dudzik E, Prokeš K, Mydosh JA, Huang Y-K, Pöttgen R. Valence Modulations in CeRuSn. Phys Rev B 2014;90:041104 (4p).

[10] Movshovich R, Lawrence JM, Hundley MF, Neumeier J, Thompson JD, Lacerda A, Fisk Z. Magnetism in CePtPb. Phys Rev B 1996;53:5465-5471.

[11] Matar SF. Review on Cerium Intermetallic Compounds: A Bird's Eye Outlook through DFT. Progr Solid State Chem 2013;41:55-85.

[12] Gupta S, Suresh KG. Review on Magnetic and Related Properties of RTX Compounds. J Alloys Compd 2015;618:562-606.

[13] Sakurai J, Kawamura R, Taniguchi T, Nishigori S, Ikeda S, Goshima H, Suzuki T, Fujita T. Transition from Antiferromagnetic Kondo Metal to Mixed Valence semiconductor in Ce( $\left.\mathrm{Pt}_{1-\mathrm{x}} \mathrm{Ni}_{\mathrm{x}}\right) \mathrm{Sn}$. J Magn Magn Mater 1992;104-107:1415-1417.

[14] Ślebarski A, Maple MB, Baumbach RE, Sayles TA. Interplay Between SpinGlass-Like and Non-Fermi Liquid Behavior in CeNi1-x $\mathrm{Rh}_{\times} \mathrm{Sn}$. Phys Rev B 2008;77:245133 (9p).

[15] Hermes W, Mishra R, Müller H, Johrendt D, Pöttgen R. Structure and Properties of Dimorphic CePdZn. Z Anorg Allg Chem 2009;635:660-666. 
[16] Svitlyk V, Hermes W, Chevalier B, Matar SF, Gaudin E, Voßwinkel D, Chernyshov D, Hoffmann R-D, Pöttgen R. Change of the Cerium Valence with Temperature - Structure and Chemical Bonding of HT-CeRhGe. Solid State Sci 2013; 21:6-10.

[17] Riecken JF, Heymann G, Soltner T, Hoffmann R-D, Huppertz H, Johrendt D, Pöttgen R. The High-Pressure Modification of CePtSn - Synthesis, Structure, and Magnetic Properties. Z Naturforsch 2005;60b:821-830.

[18] Hermes W, Chevalier B, Rodewald UCh, Matar SF, Weill F, Schellenberg I, Pöttgen R, Lueken H, Speldrich M. New Quaternary Hydride $\mathrm{CeZnSnH}_{1.5}$ Structure, Magnetism, and Chemical Bonding. Chem Mater 2011;23:1096-1104.

[19] Dhar SK, Pattalwar SM. Vijayaraghavan R. CeGeGa - A Ferromagnetic Dense Kondo System. Physica B 1993;186-188:491-493.

[20] Grin YN, Rogl P, Chevalier B, Fedorchuk AA, Gryniv IA. Physical Properties of Binary Cerium Gallides and Ternary Cerium-Germanium Gallides. J LessCommon Met 1991;167:365-371.

[21] Dhar SK, Pattalwar SM, Vijayaraghavan R. Magnetic and Thermal Behavior of $\mathrm{CeAl} X(X=\mathrm{Si}$ and Ge) Compounds. J Magn Magn Mater 1992;104-107:13031304.

[22] Mori H, Sato N, Satoh T. An Electronically-Driven Volume Transition in CeSi2xGax. Solid State Commun 1984;49:955-958.

[23] Moshchalkov VV, Petrenko OV, Zalyalyutdinov MK. The New Kondo Lattice Compounds: CeSi2-xGax. Physica B 1990;163:395-397.

[24] Kraft R, Pöttgen R, Kaczorowski D. Magnetic and Electrical Behavior in CeMgGa. Chem Mater 2003;15:2998-3002.

[25] Kraft R, Valldor M, Pöttgen R. Ternary Gallides $R E M g G a(R E=$ Y, La, Pr, Nd, Sm-Tm, Lu) - Synthesis and Crystal Chemistry. Z Naturforsch 2003;58b:827831.

[26] Kraft R, Valldor M, Kurowski D, Hoffmann R-D, Pöttgen R. Ternary Indides $R E M g I n(R E=\mathrm{Y}, \mathrm{La}-\mathrm{Nd}, \mathrm{Sm}, \mathrm{Gd}-\mathrm{Tm}, \mathrm{Lu})-$ Synthesis, Structure and Magnetic Properties. Z Naturforsch 2004;59b:513-518.

[27] Kraft R, Pöttgen R. Ternary Thallides $R E M g T l ~(R E=\mathrm{Y}, \mathrm{La}-\mathrm{Nd}, \mathrm{Sm}, \mathrm{Gd}-\mathrm{Tm}$, Lu). Z Naturforsch 2005;60b:265-270.

[28] Singh RP. First Principle Study of Structural, Electronic and Thermodynamic Behavior of Ternary Intermetallic Compound: CeMgTl. J Magnesium Alloys 2015;2:349-356.

[29] Fickenscher T, Pöttgen R. Synthesis and Crystal Structure of $R E A g M g(R E=L a$, Ce, Nd, Eu, Gd, Tb, Ho, Tm, and Yb). J Solid State Chem 2001;161:67-72.

[30] Gibson BJ, Das A, Kremer RK, Hoffmann R-D, Pöttgen R. Synthesis, Structure, and Magnetic Properties of $\mathrm{LaTMg}$ and $\mathrm{Ce} T \mathrm{Mg}(T=\mathrm{Pd}, \mathrm{Pt}, \mathrm{Au})$. J Phys $\mathrm{C}$ 2002;14:5173-5186.

[31] Johrendt D, Kotzyba G, Trill H, Mosel BD, Eckert H, Fickenscher T, Pöttgen R. Magnetic and Electrical Properties, ${ }^{151}$ Eu Mössbauer Spectroscopy and Chemical 
Bonding of $R E A g M g(R E=\mathrm{La}, \mathrm{Ce}, \mathrm{Eu}, \mathrm{Yb})$ and EuAuMg. J Solid State Chem 2002;164:201-209.

[32] Kohn W, Sham LJ. Self-Consistent Equations Including Exchange and Correlation Effects. Phys Rev 1965;140:A1133-A1138.

[33] Hohenberg P, Kohn W. Inhomogeneous Electron Gas. Phys Rev 1965;136:B864B871

[34] Kresse G, Furthmüller J. Efficient iterative schemes for ab initio total-energy calculations using a plane-wave basis set. Phys Rev B 1996;54:11169-11186.

[35] Kresse G, Joubert J. From ultrasoft pseudopotentials to the projector augmentedwave method, Phys Rev B 1999;59:1758-1775.

[36] Blöchl PE. Projector augmented-wave method. Phys Rev B 1994;50:1795317979.

[37] Perdew J, Burke K, Ernzerhof M. Generalized Gradient Approximation Made Simple. Phys Rev Lett 1996;77:3865-3868.

[38] Press WH, Flannery BP, Teukolsky SA, Vetterling WT. Numerical Recipes, Cambridge University Press, New York, 1986.

[39] Blöchl PE, Jepsen O, Andersen OK. Improved tetrahedron method for Brillouinzone integrations. Phys. Rev. B 1994, 49, 16223

[40] Methfessel M, Paxton AT. High-precision sampling for Brillouin-zone integration in metals. Phys Rev B 1989;40:3616-3621.

[41] Monkhorst HJ, Pack JD. Special points for Brillouin-zone integrations. Phys Rev B 1976;13:5188-5192.

[42] Williams AR, Kübler J, Gelatt Jr CD. Cohesive properties of metallic compounds: Augmented-spherical-wave calculations. Phys Rev B 1979;19:6094-6118.

[43] Eyert V. Basic notions and applications of the augmented spherical wave method. Int J Quantum Chem 2000;77:1007-1031.

[44] Eyert V, The Augmented Spherical Wave Method, Lect Notes Phys 849, Springer, Berlin, 2013.

[45] Hoffmann R. How Chemistry and Physics Meet in the Solid State. Angew Chem Int Ed Engl 1987;26:846-878.

[46] Krypyakevich PI, Markiv VYa, Melnyk EV. Crystal Structures of the Compounds $\mathrm{ZrNiAl}, \mathrm{ZrCuGa}$ and their Analogues. Dopov Akad Nauk Ukr RSR Ser A 1967:750-753.

[47] Dwight AE, Mueller MH, Conner RA Jr, Downey JW, Knott H. Ternary Compounds with the $\mathrm{Fe}_{2} \mathrm{P}-\mathrm{Type}$ Structure. Trans Met Soc AIME 1968;242:2075-2080. 
[48] Zumdick MF, Hoffmann R-D, Pöttgen R. The Intermetallic Zirconium Compounds $\mathrm{ZrNiAl}, \mathrm{ZrRhSn}$, and $\mathrm{ZrPtGa}$ - Structural Distortions and Metal-Metal Bonding in $\mathrm{Fe}_{2} \mathrm{P}$ Related Compounds. Z Naturforsch 1999;54b:45-53.

[49] Donohue J. The Structures of the Elements, Wiley, New York, 1974.

[50] Emsley J. The Elements, Clarendon Press, Oxford; 1989.

[51] Birch F. Finite strain isotherm and velocities for single-crystal and polycrystalline $\mathrm{NaCl}$ at high pressures and $300 \mathrm{~K}$. J Geophys Res 1978;83:1257-1268.

[52] Bader RFW. A quantum theory of molecular structure and its applications. Chem Rev 1991;91:893-928. 
Table 1

Lattice parameters of equiatomic compounds $\operatorname{LaMg} X$ and $\operatorname{CeMg} X(X=$ $\mathrm{Ga}, \mathrm{In}, \mathrm{Tl}, \mathrm{Pd}, \mathrm{Ag}, \mathrm{Pt}, \mathrm{Au}$ ) with hexagonal $\mathrm{ZrNiAl}$ type structure.

\begin{tabular}{|c|c|c|c|c|c|}
\hline Compound & $a / \mathrm{pm}$ & $c / \mathrm{pm}$ & $V / \mathrm{nm}^{3}$ & $c / a$ & Reference \\
\hline \multicolumn{6}{|c|}{ triel intermetallics } \\
\hline $\mathrm{LaMgGa}$ & $760.2(2)$ & $459.6(1)$ & 0.2300 & 0.605 & [25] \\
\hline LaMgIn & $782.6(2)$ & $481.08(9)$ & 0.2552 & 0.615 & [26] \\
\hline LaMgT1 & $781.3(1)$ & $477.84(8)$ & 0.2526 & 0.612 & [27] \\
\hline $\mathrm{CeMgGa}$ & $752.7(2)$ & $454.8(1)$ & 0.2232 & 0.604 & [25] \\
\hline CeMgIn & $774.9(3)$ & $477.7(2)$ & 0.2484 & 0.616 & {$[26]$} \\
\hline CeMgTl & $774.1(1)$ & $473.75(7)$ & 0.2458 & 0.612 & [27] \\
\hline \multicolumn{6}{|c|}{ transiton metal intermetallics } \\
\hline $\mathrm{LaMgPd}$ & $773.6(1)$ & $413.79(4)$ & 0.2145 & 0.535 & {$[30]$} \\
\hline LaMgAg & $788.8(3)$ & $437.4(1)$ & 0.2357 & 0.555 & [29] \\
\hline $\mathrm{LaMgPt}$ & $762.0(1)$ & $417.48(5)$ & 0.2099 & 0.548 & [30] \\
\hline $\mathrm{LaMgAu}$ & $781.0(1)$ & $425.49(9)$ & 0.2248 & 0.545 & [30] \\
\hline CeMgPd & $767.3(1)$ & $410.37(4)$ & 0.2092 & 0.535 & {$[30]$} \\
\hline $\mathrm{CeMgAg}$ & $782.5(3)$ & $432.8(1)$ & 0.2295 & 0.553 & [29] \\
\hline $\mathrm{CeMgPt}$ & $755.02(7)$ & $413.82(4)$ & 0.2043 & 0.548 & {$[30]$} \\
\hline $\mathrm{CeMgAu}$ & $774.1(3)$ & $421.6(1)$ & 0.2188 & 0.545 & [30] \\
\hline
\end{tabular}

Table 2

Interatomic distances (pm), within the first coordination spheres of CeMgIn, $\mathrm{CeMgTl}, \mathrm{CeMgAg}$, and CeAuMg.

\begin{tabular}{|c|c|c|c|c|c|c|c|c|c|c|c|c|c|}
\hline \multicolumn{3}{|c|}{ CeMgIn [26] } & \multicolumn{3}{|c|}{ CeMgTl [27] } & \multicolumn{4}{|c|}{ CeMgAg [29] } & \multicolumn{4}{|c|}{ CeAuMg [30] } \\
\hline Ce: 4 & In1 & 331.5 & Ce: 1 & $\mathrm{Tl} 2$ & 328.5 & Ce: & 4 & $\mathrm{Ag} 1$ & 319.7 & Ce: & 4 & Au1 & 314.2 \\
\hline 1 & In2 & 334.6 & 4 & Tl1 & 330.9 & & 1 & $\mathrm{Ag} 2$ & 325.4 & & 1 & $\mathrm{Au} 2$ & 321.2 \\
\hline 2 & $\mathrm{Mg}$ & 347.3 & 2 & $\mathrm{Mg}$ & 349.4 & & 2 & $\mathrm{Mg}$ & 345.4 & & 2 & $\mathrm{Mg}$ & 339.7 \\
\hline 4 & $\mathrm{Mg}$ & 376.1 & 4 & $\mathrm{Mg}$ & 371.0 & & 4 & $\mathrm{Mg}$ & 356.2 & & 4 & $\mathrm{Mg}$ & 350.0 \\
\hline 4 & $\mathrm{Ce}$ & 398. & 4 & $\mathrm{Ce}$ & 400 & & 4 & $\mathrm{Ce}$ & 6 & & 4 & $\mathrm{Ce}$ & 03.5 \\
\hline 2 & $\mathrm{Ce}$ & 477.7 & 2 & $\mathrm{Ce}$ & 473 & & 2 & $\mathrm{Ce}$ & 8 & & 2 & $\mathrm{Ce}$ & 21.6 \\
\hline In1: 3 & $\mathrm{Mg}$ & 299.6 & T11: 3 & $\mathrm{Mg}$ & 298.7 & Ag1: & 3 & $\mathrm{Mg}$ & 303.9 & Au1: & 3 & $\mathrm{Mg}$ & 300.2 \\
\hline 6 & $\mathrm{Ce}$ & 331. & 6 & $\mathrm{Ce}$ & 330.9 & & 6 & $\mathrm{Ce}$ & & & 6 & $\mathrm{Ce}$ & 314.2 \\
\hline In2: 6 & $\mathrm{Mg}$ & 304.1 & T12: 6 & $\mathrm{Mg}$ & 302.9 & Ag2: & 6 & $\mathrm{Mg}$ & 286.6 & $\mathrm{Au} 2:$ & 6 & $\mathrm{Mg}$ & 281.5 \\
\hline 3 & $\mathrm{Ce}$ & 334.6 & 3 & $\mathrm{Ce}$ & 328.5 & & 3 & $\mathrm{Ce}$ & 325.4 & & 3 & $\mathrm{Ce}$ & 321.2 \\
\hline Mg: 2 & In1 & 299.6 & Mg: 2 & Tl1 & 298.7 & Mg: & 2 & Ag2 & 286.6 & Mg: & 2 & $\mathrm{Au} 2$ & 281.5 \\
\hline 2 & In2 & 304 & 2 & $\mathrm{~T} 12$ & 302 & & 2 & $\mathrm{Ag} 1$ & 30 & & 2 & Au1 & 300.2 \\
\hline 2 & $\mathrm{Mg}$ & 325. & 2 & $\mathrm{Mg}$ & 327 & & 2 & $\mathrm{Mg}$ & 32 & & 2 & $\mathrm{Mg}$ & 323.1 \\
\hline 2 & $\mathrm{Ce}$ & 347.3 & 2 & $\mathrm{Ce}$ & 349.4 & & 2 & $\mathrm{Ce}$ & 345.4 & & 2 & $\mathrm{Ce}$ & 339.7 \\
\hline 4 & $\mathrm{Ce}$ & 376.1 & 4 & $\mathrm{Ce}$ & 371.0 & & 4 & $\mathrm{Ce}$ & 356.2 & & 4 & $\mathrm{Ce}$ & 350.0 \\
\hline
\end{tabular}


Table 3

Calculated parameters of equiatomic compounds $\operatorname{LaMg} X$ and $\operatorname{CeMg} X(X=\mathrm{Ga}, \mathrm{Pd})$ : Total and cohesive energies per unit cell, volumes and bulk moduli are in $\mathrm{eV}, \mathrm{nm}^{3}$ and $\mathrm{GPa}$ units respectively.

\begin{tabular}{lcccc}
\hline Compound & $E_{\text {total }}$ & $E_{\text {cohesive }}$ & Vexp.(calc.) & $\mathrm{B}_{0}$ \\
\hline LaMgGa & -32.36 & -1.56 & $0.230(0.232)$ & 49.8 \\
CeMgGa & -31.77 & -1.86 & $0.223(0.229)$ & 51.4 \\
& & & & \\
LaMgPd & -40.87 & -2.13 & $0.214(0.218)$ & 60.5 \\
CeMgPd & -40.11 & -2.38 & $0.209(0.215)$ & 62.3 \\
\hline
\end{tabular}

Energies per atom of constituents: $E(\mathrm{Ce})=-4.43 \mathrm{eV} ; E(\mathrm{La})=$ $4.92 \mathrm{eV} ; E(\mathrm{Ga})=-2.91 \mathrm{eV} ; E(\mathrm{Mg})=-1.39 \mathrm{eV} ; E(\mathrm{Pd})=-5.17$ $\mathrm{eV}$

\section{Table 4}

Calculated parameters of equiatomic compounds $\operatorname{LaMg} X$ and $\mathrm{CeMg} X(X=\mathrm{Ga}, \mathrm{Pd})$ : Bader charges per atom in $e$ units.

\begin{tabular}{lcccc}
\hline Compound & $Q(\mathrm{La} / \mathrm{Ce})$ & $Q(\mathrm{Mg})$ & $Q(X 1)$ & $Q(X 2)$ \\
\hline $\mathrm{LaMgGa}$ & 1.05 & 1.13 & -2.53 & -2.01 \\
$\mathrm{CeMgGa}$ & 1.07 & 1.14 & -2.03 & -2.56 \\
& & & & \\
$\mathrm{LaMgPd}$ & 1.16 & 1.14 & -2.86 & -2.02 \\
$\mathrm{CeMgPd}$ & 1.19 & 1.15 & -2.87 & -2.08 \\
\hline
\end{tabular}

Table 5

Calculated parameters of equiatomic $\mathrm{CeMgGa}$ and $\mathrm{CeMgPd}$ in ferromagnetic configurations (not ground state). Energies are in eV/cell and Ce magnetic moments (spin-only) in $\mu_{\mathrm{B}}$.

\begin{tabular}{lcccc}
\hline $\mathrm{CeMg} X$ & $\Delta E$ SPF-NSP & $m_{C e}$ & $m_{M g / X I / X 2}$ & $M($ cell $)$ \\
\hline$X=\mathrm{Ga}$ & -0.176 & 0.841 & $0.04 / 0.004 / 0.01$ & 2.674 \\
$X=\mathrm{Pd}$ & -0.046 & 0.595 & $0.02 / 0.019 / 0.02$ & 1.922 \\
\hline
\end{tabular}




\section{FIGURE CAPTIONS}

Fig. 1. Projection of the $\operatorname{CeMg} X$ structure onto the $x y$ plane. Cerium, magnesium, and $X$ atoms are drawn as medium gray, black open, and filled circles, respectively. All atoms lie on mirror planes at $z=0$ (thin lines) and $z=1 / 2$ (thick lines). The trigonal prisms around the $X$ atoms are emphasized.

Fig. 2. Triel intermetallics with $X=$ Ga representatives in non magnetic total spin configuration (NSP). CeMgGa (top) and $\mathrm{LaMgGa}$ (bottom).

Fig. 3. Transition metal intermetallics with $X=\mathrm{Pd}$ representatives in non magnetic total spin configuration (NSP). CeMgPd (top) and LaMgPd (bottom).

Fig. 4. Site and spin projected density of states (DOS) in SPF configuration of CeMgGa (top) and CeMgPd (middle) and SPAF total DOS for SPAF CeMgGa (bottom).

Fig. 5. Chemical bonding for $X=\mathrm{Ga}$ (top) and $X=\mathrm{Pd}$ (bottom) in $\operatorname{CeMg} X$ and $\operatorname{LaMg} X$. 


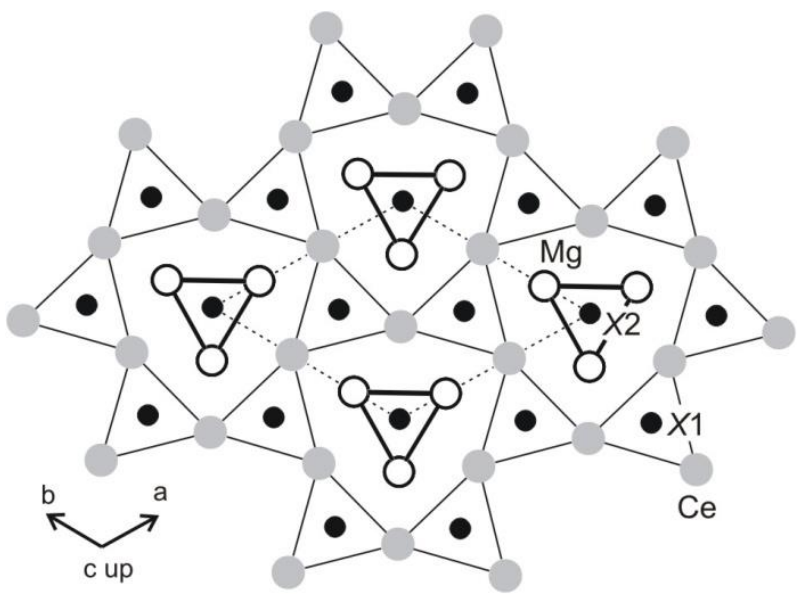

Fig. 1. 

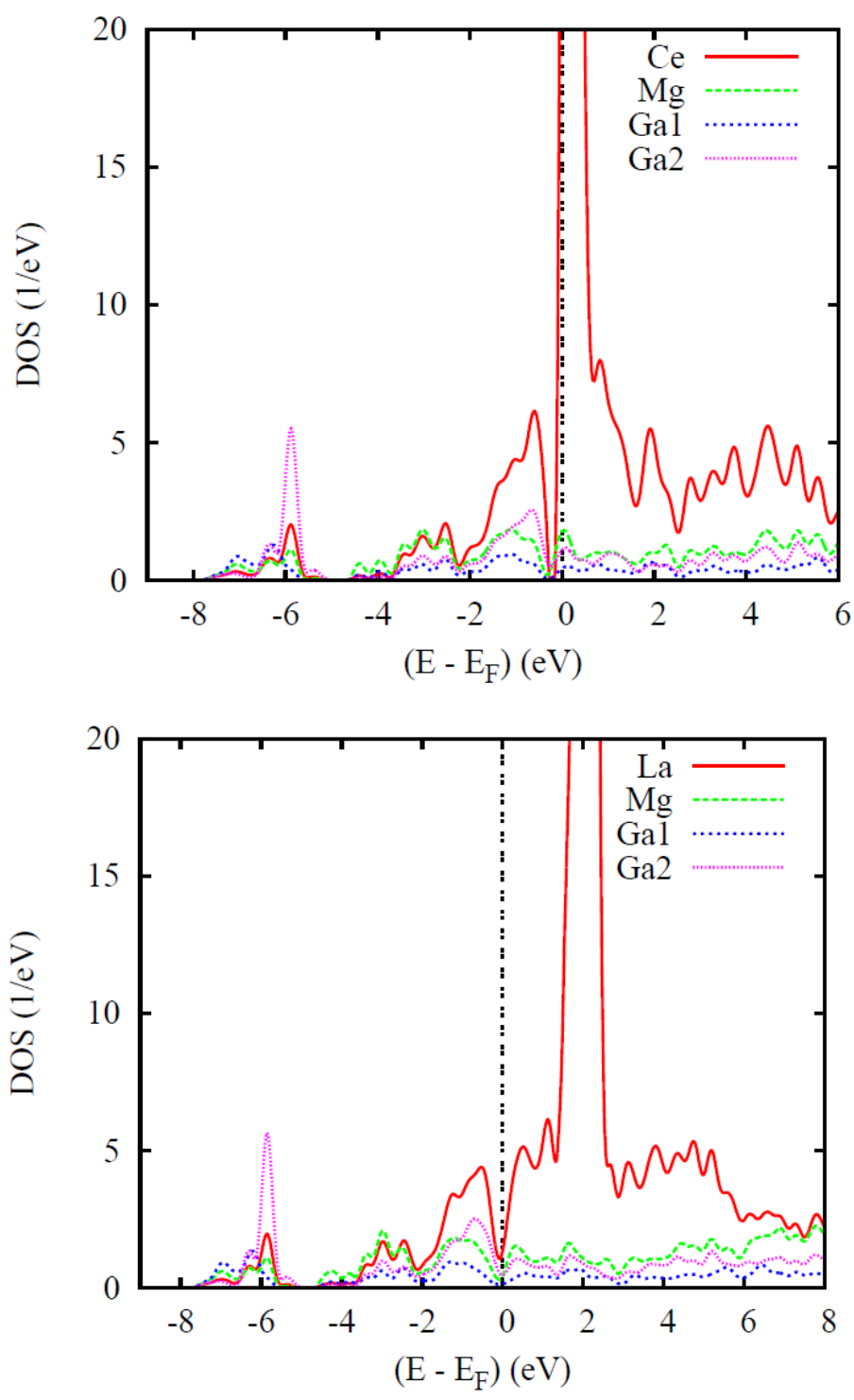

Fig. 2. 

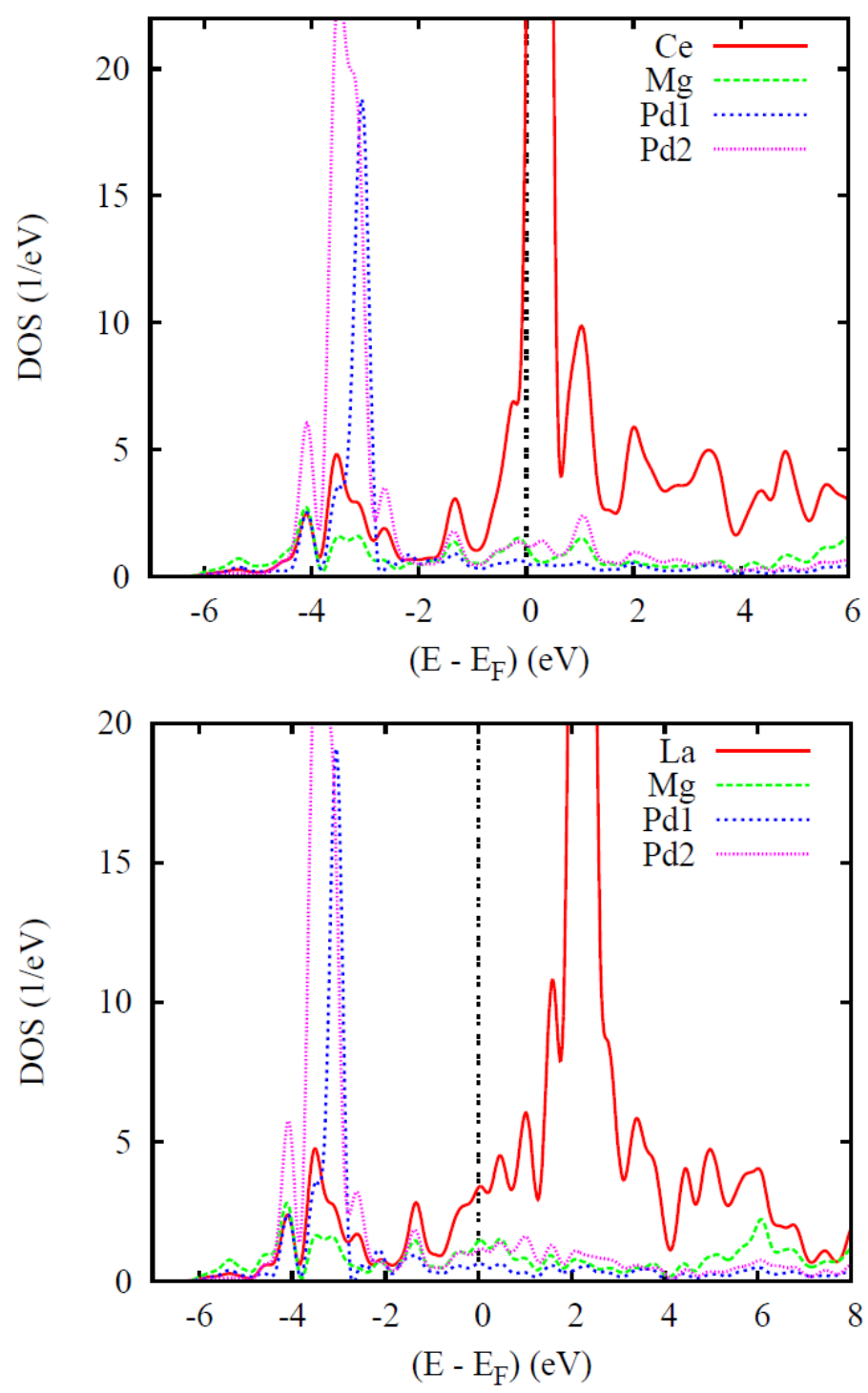

Fig. 3. 

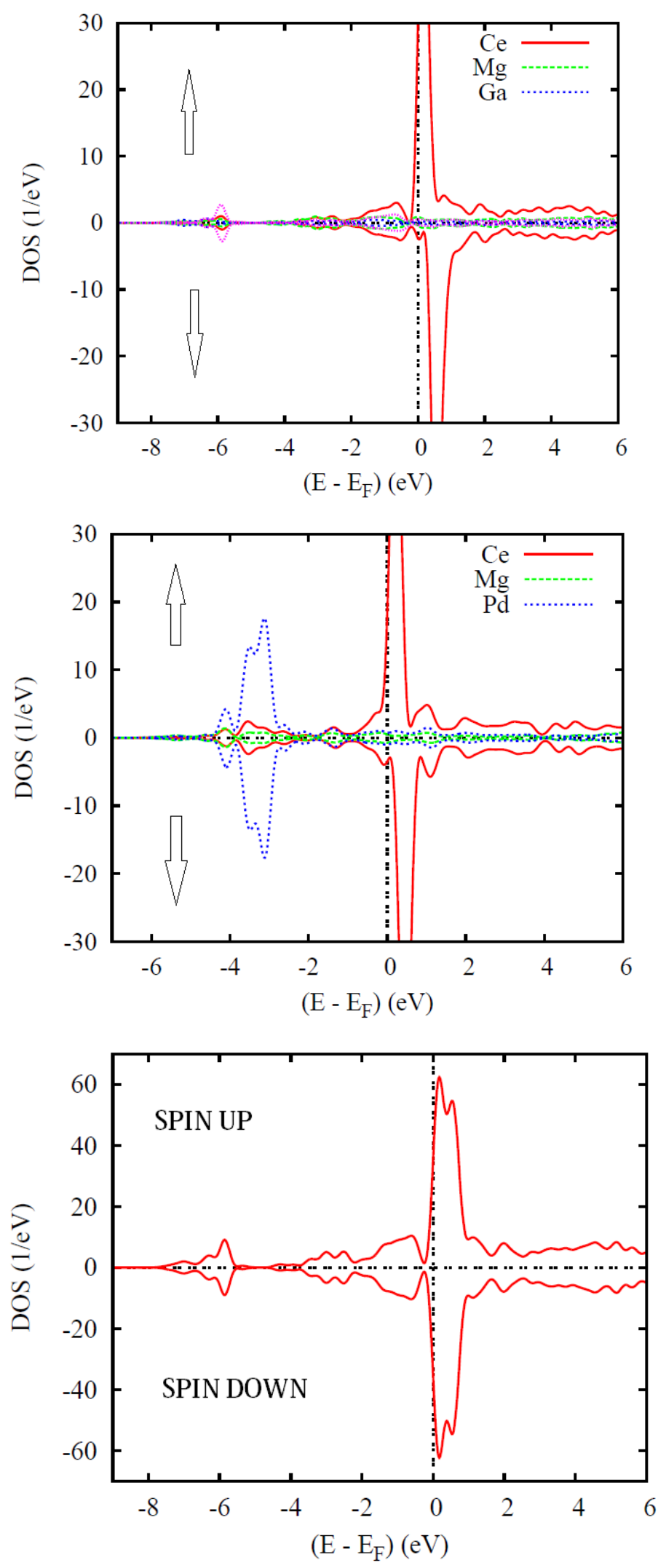

Fig. 4. 

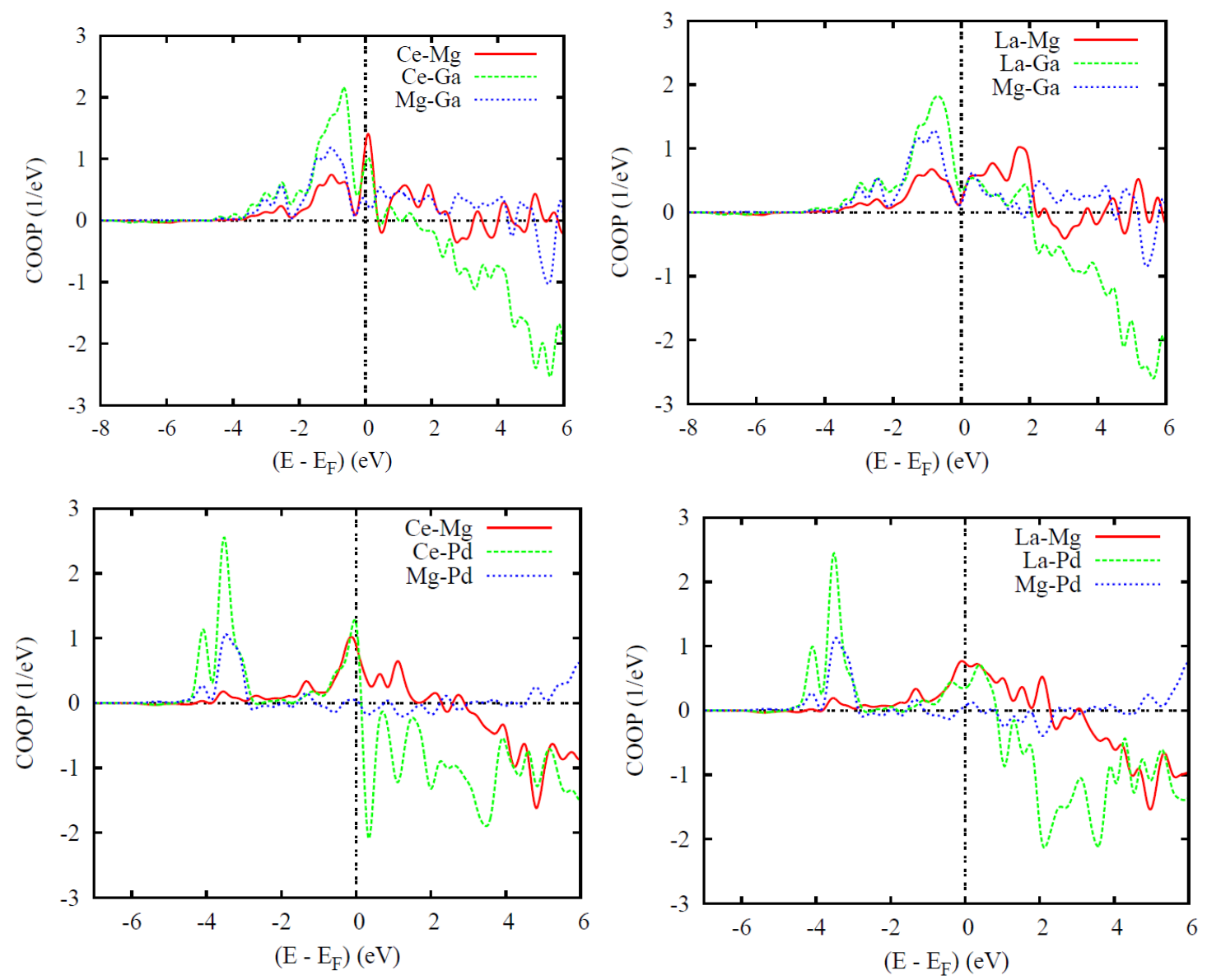

Fig. 5 